\title{
Identification and Characterization of Traffic Flow Patterns for UTM application
}

\author{
Abdulrahman Alharbi \\ School of Aerospace, Transport and \\ Manufacturing \\ Cranfield University \\ Cranfield, UK \\ Abdulrahman.A.Alharbi@cranfield.ac.uk
}

\author{
Ivan Petrunin \\ School of Aerospace, Transport and \\ Manufacturing \\ Cranfield University \\ Cranfield,UK \\ i.petrunin@cranfield.ac.uk
}

\author{
Dimitrios Panagiotakopoulos \\ School of Aerospace, Transport and \\ Manufacturing \\ Cranfield University \\ Cranfield,UK \\ D.panagiotakopoulos@cranfield.ac.uk;
}

\begin{abstract}
The current airspace has limited resource, and the widespread use of Unmanned Aircraft System (UAS) is increasing the density of civilian aircraft that is already crowded with manned aerial vehicles. This increased density in airspace demands to improve the safety, efficiency and capacity of airspace while considering all uncertain parameters that may cause hinderance in aircraft movement like weather and dynamic fluctuations. A systematic analysis of correlations between events and their impacts in air traffic network is a considerable challenge. This paper proposes a methodology that characterizes and identifies the patterns of Unmanned Traffic Management (UTM) airspace based on the analysis of simulated data to improve the performance of UTM network as well as ensuring its safety and capacity. Some sets of metrics are defined to identify the airspace characteristics that include airspace density, capacity and efficiency. The data analysis carried out here, will support risk analysis and improve trajectory planning in different airspace regions considering all dynamic parameters such as extreme weather conditions, loss of safe distances, UAVs' performance, emergency services and airspace structures that may cause deviations from their standard paths.
\end{abstract}

Keywords- UAV, traffic flows patterns, trajectory deviation, simulation, UTM.

\section{I.INTRODUCTION}

Unmanned Aaerial Vehicle (UAV) presents an immense opportunity to commercial and industrial activities due to its robustness and flexibility. The global market for UAVs is predicted to expand at a rate of $15.88 \%$ till 2026 [1] and it is anticipated that demand for different services such as delivery, surveillance, and aerial photography will be increased. With its increasing demand, it has become necessary to develop such an adroit traffic management system for UAV operations in urban areas that would be smart and optimized.

The concepts of Unmanned Traffic Management (UTM) [2] and Urban Air Mobility (UAM) [3] have been proposed in above studies to carry out a safe and efficient aerial vehicle operations. It is emphasized in the above studies that UTM and UAM systems have changed the paradigm of existing air traffic systems and posed challenges to aircraft safety and efficiency due to a variety of factors including various vehicle characteristics; increased in density of vehicles' operations, urban terrain environments, and complex atmospheric conditions in lower airspace. A question thus arises; How to handle high density operations safely and efficiently under these challenging situations?

Also, the demand for UAM is also gaining interest due to increased road traffic in metropolitan areas where the requirement to transport passengers and cargo safely and efficiently in the urban areas [3] is increasing. This will also pose more challenges on safety and efficiency of these UAV systems, and capacity of the UTM. Also, for large scale urban operations, safety and efficiency are always a top priority.

Although, a number of studies on the complexity and capacity of conventional Air Traffic Management (ATM) systems have been conducted [4] and some initial studies on small UAS operations have been done[5], yet the understanding of key factors that affect the high density of UAM and UTM operations is still limited. Thus, there is a need to understand the provision of safe and efficient high-density air traffic operations under extreme weather conditions analytically.

Since, efficiency and safety of airspace dictate the capacity which is also influenced by traffic flow behaviour, several studies have applied advance analytical methods to use flight trajectory data in order to characterize the air traffic behaviour towards supporting performance assessment, airspace monitoring, airspace design and traffic flow management [6]. A study in [7] focussed on detection and identification of significant events where a large data is produced every day by stake holders of ATM systems using data analytics. These techniques may help pointing out capacity indicators, extracting safety models, risk identification and decision support for better ATM systems. Although, ATM domain is rich in such studies, limited studies have been made in the UTM context. The reason behind this, is a lack of research publications and more focus is on implementation and control approaches of UAVs [8]. Moreover, a considerable barrier in data analysis of UTM is the absence of a common and shared database collecting real-time UAV flight operation data. One possible development direction is to use simulation data [9].

The rest of the paper is organized as follows: Section II presents related work and it provides some background on the techniques used in this paper. Section III presents the proposed methodology of this work. Section IV presents the simulation scenarios and result .Section $V$ shows the airspace performance

(C) 2021 IEEE. Personal use of this material is permitted. Permission from IEEE must be obtained for all other uses, in any current or future media, including

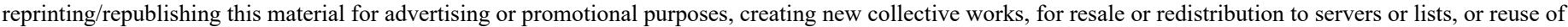
any copyrighted component of this work in other works 
analysis. Lastly, Section VI draws the conclusions of this paper and provides some guidelines for future work.

\section{RELATED WORK}

The significant increase in air vehicle traffic results in congestion in the airspace. This congestion is a critical issue to be dealt with, in the development of new and improved air traffic management systems such as UTM [10]. UAV autonomous operations are currently vital for the development of several civilian and military applications enabling safe navigation with little or no human supervision [11]. UTM is imagined as a subset of ATM that enables safe and efficient low-altitude airspace operations by providing services such as airspace design and dynamic configuration, dynamic geo-fencing, severe weather and wind avoidance, congestion management, terrain avoidance, route planning and re-routing, separation management, sequencing and spacing, and contingency management [12].

The primary issue in such mission-oriented UAVs, is to maintain path plans and minimum separation distance among UAVs and other static and dynamic obstacles [13].

The work in [14] highlighted the importance of addressing the weather conditions that may pose uncertain situations to UTM such as potential delay in UAVs, UAV conflicts, etc. According to them most common issues that may need to be handled includes predicting UAV stability and adapting it to weather conditions such as crosswinds, unpredicted rains, avoiding collisions, and solving conflicts that may arise during flight. Similarly, the study in [15] enlighten the significance of disruptions in schedules and flight plans caused by bad weather that is a challenge for air traffic management systems, due to uncertain behaviour of weather systems.

It is well understood that the uncertainty in weather and conflict resolution strategies incorporate the deviation in planned trajectory patterns thus, threatening airspace capacity, it is also needed to visualize the capacity of UTM airspace in this context. A significant research area of knowledge discovery and information extraction involves detection and identification of events present in historical aircraft trajectory system. This research area focuses on extraction of valuable information from air traffic management system. The extracted event-related information can help stakeholders in different respects such as capacity indicators, extracting safety models and risk estimation [7]. Such knowledge discovery systems are valuable in detecting, identifying and characterizing air traffic flows as narrated in [16].

The recent success of data-analytic techniques in the areas of perception, planning, and control make it suitable for conflict detection and resolution in the UAV network. Moreover, ATM generates vast amounts of flight data from routine monitoring on daily basis. However, the concept of extracting safety knowledge from this data is still based on detecting the boundaries of expert-defined thresholds[17]. The volume of data generated by a multi-UAV mission grows and may exceed the attention capability of the operator[18]. Data analytical techniques present the theories, technologies and processes of acquiring in-depth knowledge from the historical data.
The work in[19] narrated the applications of data analytics in different aspects of air traffic management that include conflict detection, delay reduction, environmental impact mitigation, flight efficiency and safety enhancement. Büsing et al. [20] employed statistical data analysis methods to the data of Lufthansa German Airlines revenue management system. They examined revenue management models and algorithms in airlines considering the capacity uncertainty caused by fleetassignment-optimizations.

They concluded that $40 \%$ of their flights fleets assignment is updated once in the booking horizon. The findings of their work demonstrate that flight results can be improved by ignoring traditional fixed capacity assumption. Similarly, Chung et al. [21] also analysed flight delays data of an airline in Hong Kong. They observed that there is a direct correlation between flight arrival delays and departure delays. Therefore, they proposed a new cascading neural network for extracting the identified characteristics. In the light of above literature review, the aim of this paper is to address the UTM airspace capacity in two folds: Firstly, the efficiency and safety of the UTM airspace is measured in the presence of abnormal events specifically during weather uncertainties. Secondly, the traffic flow behaviours have been observed in weather hazards by studying spatial and temporal recurrent and concurrent traffic flow patterns due to trajectory deviations and their impacts on the capacity of UTM airspace. To that end, this paper contributes in the following ways:

1. Developed a simulating based experimental framework for trajectory data generation for both fixed-wing and rotary-wing type UAVs under varying weather and dynamical constraints and priority levels.

2. Studied the effects of weather conditions and emergency operations on the safety, capacity and efficiency of UTM airspace airspace using proposed metrics of safety, density, capacity, time and energy efficiencies.

3. Visualized different types of traffic flow patterns and studied trajectory deviations and their role in reducing the safety, capacity and efficiency of the UTM airspace.

\section{METHODLOGY}

\section{A. Simualtion Setting and Consideration}

The proposed method is assessed and validated through simulations that simulate the airspace over Bedfordshire and Buckinghamshire in the UK. It defines seven areas where flights may be restricted such as airfields, recreational areas and a prison as illustrated in Fig. 1. These ares are:

1. Two airfields, including Luton and Cranfield (Orange);

2. Four recreational areas, including Dunstable, Sandy, Cardington and Graveley (Yellow);

3. Milton Keynes Prison (Blue). 


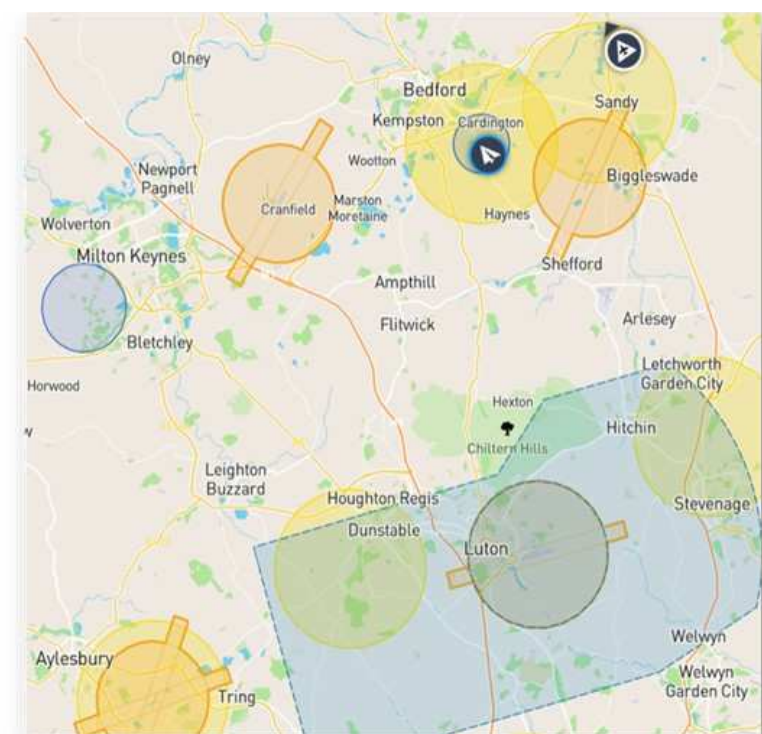

Fig. 1.Simulation environment created by Air Map

The following simulation settings are considered that set the limitations, requirements, and make things more realistic and closer to a real-life scenario:

- 2D airspace: Due to the current limitation of our simulation framework of not simulating ascend and descend manoeuvres, the current work is visualizing the cruise phase of UAVs in 2D (x, y) only.

- Hybrid fixed and random start and end locations: The simulation parameters have been set in order to make the UAV flight start and finish locations fixed as well as random to simulate normal daily services as well as emergency services.

- Random departure times: To make the system more realistic, the exact time at which each agent departs is set randomly between 1-10 minutes in 1 hour of simulation scenario period.

- Priority levels: Each flight has been set with a priority level of service i.e.; from level 1 (highest priority) to level 5 (lowest priority). based on urgency. The description of each priority level is as follows:(1): lifesaving activities, (2): national security, (3) life Support, (4) All other activities.

- UAV types: this simulation considers two basic type of UAVs mainly: fixed wing and rotary to consider the effects of their structural dynamics.

\section{B. Weather Factors Implementation}

Weather hazards considered for UAVs operations are classified as moderate, adverse and severe [15]. Here, moderate hazards are those that result from phenomena that reduce visibility but do not harm the aircraft. Adverse hazards are those weather conditions that have potential to cause loss of control, loss of communication, diminish aerodynamic performance and may negatively affect the operator. Lastly, severe hazards are those which would result in severe damage or loss control of an aircraft.

\section{- Wind impact on UAVs operation:}

This is a widespread phenomenon that wind causes a change in the direction of UAV when its direction is normal to the direction of UAV [22]. Strong winds have capability to affect the ground speed and flight path of an unmanned aircraft. Similar to larger manned aircraft, headwinds and tailwinds, decrease or increase the ground speeds. Unlike typical manned aircraft, wind speeds can easily surpass the maximum speeds of the UAVs. Generally, rotary-wing aircrafts have slower maximum speeds than those of fixed-wing aircrafts. The aero dynamical structure of fixed wing UAVs helps the aircraft to maneuver through air streamlines whereas rotary-wing UAVs are more likely to struggle even at lower wind speed [15].

Wind impact on UAV operations can be presented mathematically as following:

$$
\begin{aligned}
& l_{1}=\operatorname{Tan} \varphi_{1} \\
& l_{2}=\frac{y(k+1)-y(k)}{x(k+1)-x(k)} \\
& \operatorname{Tan} \varphi_{2}=\frac{\left(l_{2}-l_{1}\right)}{\left(1+\left(l_{2}\right)\left(l_{1}\right)\right)} \\
& x=\cos \varphi_{2} \\
& y=\sin \varphi_{2}
\end{aligned}
$$

In the above set of equations used $\varphi_{1}$ represents the direction of wind and $\varphi_{2}$ represents the direction of deviations in UAVs caused by wind. Discretized steps of the simulation are represented by $\mathrm{k}$. As it is known, when UAVs interact with wind direction at every step, a vertical line is formed at that instant. The $l_{1}$ and $l_{2}$ are slopes of these two vertical line equations. The $\mathrm{x}$ and $\mathrm{y}$ give the change in direction along $\mathrm{x}$-axis and $\mathrm{y}$-axis.

\section{- Rain Impact on UAVS Operation:}

The deviations caused in UAVs waypoints from planned trajectory due to rainfall can be considered by looking into the weight of water on the UAVs wings due to rain, with rotary UAVs like on a quadcopter, this effect is not that strong [15] . When UAVs fly through moderate rain the value of precipitation at each $\mathrm{cm}^{2}$ area is low. This less amount of water flows down from the UAVs wings without causing any weight addition in the accumulative weight of the UAVs body and rain water. Whereas if the rain precipitation is high, the amount of rain coming onto the UAVs wings $\mathrm{cm}^{2}$ area cause unbalance in the left side's wing weight and right side's wing weight. The unbalance of weight can be expressed as given (6) below:

$$
W_{\text {wing }}=W_{\text {str }}+W_{\text {rain }}
$$

$W_{\text {wing }}$ is representing the accumulative weight of UAVs wing $W_{\text {str }}$ structure weight and $W_{\text {rain }}$ weight of water on the wings due to rain. The weight of water on each UAVs wing can be modelled as given in (7) below:

$$
W_{\text {rain }}=W_{\text {pre }}-W_{f d}
$$

In the above equation $W_{\text {pre }}$ represents the total weight of water on each wing of the UAV and $W_{f d}$ represents the weight of water flows down from the UAVs wings due to curve and slippery structure of UAVs' wings. 
The deviations of waypoints from the planned trajectory caused by rain precipitation is directly proportional to the wings area of the UAVs. This is why rotary-wing UAVs show more resistance to the deviations as compared to fixed wing UAVs when subjected to same amount of rain precipitation.

The Table 1 shows all three-different weather classification compared to Beaufort wind scale and rainfall precipitation.

As the map has been scaled with a ratio of 1:4.3 km that can be written as:

0.006 units as the large impact $=0.006 \times 4.3=0.0258 \mathrm{~km}=25.8$ $\mathrm{m}$

0.004 units as the small impact $=0.004 \times 4.3=0.0172 \mathrm{~km}=17.2$ $\mathrm{m}$

\begin{tabular}{|l|l|l|l|}
\hline Weather type & Moderate & \multicolumn{1}{c|}{ Adverse } & Severe \\
\hline Wind $(\mathrm{m} / \mathrm{s})$ & Below 13 & $14-24$ & Above 34 \\
\hline Rainfall $(\mathrm{mm})$ & $0-2.5$ & $2.5-7.5$ & Above 7.5 \\
\hline
\end{tabular}

Table 1:Scale of weather classification used in this study

\section{Types of $U A V S$}

Current UAVs or drones may be generally classified as fixedwing or rotary-wing aircrafts. Fixed-wing UAVs are more efficient, have longer ranges and greater payloads however, rotary-wing drones take off and land vertically with the ability to hover during flight [23]. These two basic types of UAVs are considered in this simulation. The properties for fixed-wing and rotary-wing are slightly different as shown in Table 2.

\begin{tabular}{|c|c|c|}
\hline Item & Delair UX11 & Phantom 4 Pro \\
\hline UAV type & Fixed wing & Rotary-wing \\
\hline Aircraft weight & $1.6 \mathrm{~kg}$ & $1.375 \mathrm{~kg}$ \\
\hline Flight time & 52 minutes & $30 \mathrm{minutes}$ \\
\hline Cruise Speed & $54 \mathrm{~km} / \mathrm{h}$ & $50 \mathrm{~km} / \mathrm{h}$ \\
\hline Wind resistance & $45 \mathrm{~km} / \mathrm{h}$ & $10 \mathrm{~m} / \mathrm{s}$ \\
\hline
\end{tabular}

Table 2:Comparison of two UAV's types

\section{Path Planning and Optimization}

In this paper, the trajectory optimization is investigated using PSO algorithm. PSO is a population-based stochastic optimization method inspired by the behaviour of wildlife in groups such as flocks of birds or schools of fish [24]. The main advantages of PSO are that it is simple to understand, easy to implement and converges rapidly as compare to other traditional global optimization methods such as genetic algorithms and simulated annealing [25]. In addition, PSO can be leveraged to count and determine the optimal values of trajectories from A to B. In doing so, PSO identifies the best possible trajectory which is cost-effective and efficient against any challenge. The PSO algorithm is used for anticipating and planning drone path and is helpful particularly to identify the solution that requires the minimum movement between starting and end-points while avoiding any obstacle.

\section{E. Deconflicting Strategy}

A conflict is defined when two or more UAVs are in the same point at the same time. To resolved any UAV conflict that appears in the scenarios, some strategies of deconfliction have been discussed in the literature [26]. Ground delay and slow speed have been used as deconflicting strategies in our simulation setting.

\section{F. Metrics used to Characterize Airspace Traffic}

\section{- Safety}

Safety metrics focus on the ability of an airspace to affirm the safe separation among aircrafts. In traffic management, safety can be defined in terms of Loss of Separation (LoS) [27]. Separation among aircrafts is measured in terms of number of conflicts and intrusions of aircraft into other aircraft domain. In context of trajectories, Euclidean distance is used to define the closeness between two trajectories belonging to two different UAVs [28] by comparing it with a minimum separation distances. The value of the minimum safe separation distance may depend on the density of air traffic in particular region of the airspace. However, there is no pre-defined standard separation distance for UAVs, although $50 \mathrm{~m}$ is a value commonly used in research [29]. The main goal of safety metrics is to acquire Euclidean distances that violate different loss of separation levels thus deterring the safety of the UTM airspace.

Following are the details of this metrics:Let us assume two different trajectories $T_{i}$ and $T_{j}$ :

$$
\begin{aligned}
T_{i} & =\left(x_{i 1}, y_{i 1}, x_{i 2}, y_{i 2}, \ldots \ldots \ldots, x_{i n}, y_{i n},\right) \\
T_{j} & =\left(x_{j 1}, y_{j 1}, x_{j 2}, y_{j 2}, \ldots \ldots \ldots, x_{j n}, y_{j n},\right)
\end{aligned}
$$

where $x_{i n}$ is value of feature $\mathrm{n}$ for trajectory $i$ and $x_{j n}$ is value of feature $\mathrm{n}$ for trajectory $j$. Euclidean distance is used to determine the closeness between flight trajectories as follows:

$$
\begin{aligned}
& E_{d}\left(\boldsymbol{T}_{i}, \boldsymbol{T}_{j}\right)=\left\|\boldsymbol{T}_{i}-\boldsymbol{T}_{j}\right\|_{2}= \\
& \sqrt{\sum_{k=1}^{n}\left(x_{i k}-x_{j k}\right)^{2}+\left(y_{i k}-y_{j k}\right)^{2}}
\end{aligned}
$$

In order to deal a practical scenario, the trajectory of each UAV is discretized in small steps on spatial-temporal scale for measuring the Euclidean distance discussed as follows. The Euclidean distance between any two UAVs out of $\mathrm{n}$ umber of UAVs flying in a region within a specified time slot is calculated by taking a convolution between a cuboid window of $x-y-t$ axis having dimensions $(1$ unit $\times 1$ unit $\times 4 \mathrm{~min})$ and UAVs distribution on the whole $x-y-t$ airspace. By incorporating the time window as the third axis helps to realize the actual concurrence of UAVs in a region during flight time. The detected regions of UAVs trajectories within the sampling window are recorded and discretized into small steps. Euclidean distances are thus calculated by using discretized $\mathrm{x}$ and $\mathrm{y}$ coordinates of the detected trajectories.

\section{- Density}

Density of airspace is measured as the number of UAVs per unit area $(\mathrm{kmSq})$. This metric is defined as the number of UAVs in a $\mathrm{W} \times \mathrm{W}$ region across the airspace. It is calculated by using allpass filter by convolving a $\mathrm{W} \times \mathrm{W}$ window with in the entire airspace. 


$$
D(x, y)=\sum_{i, j=0}^{W-1} d(x * S+i, y * S+j)
$$

Where $d(x, y)$ gives the number of UAS at the location $(\mathrm{x}, \mathrm{y})$ and $\mathrm{S}$ is the stride size of the convolution. We refer to the matrix $D(x, y)$ as the density map and the matrix $d(x, y)$ as the distribution map [30].

\section{- Capacity}

The capacity of airspace is defined by Krozel et al. as "the maximum numbers of aircrafts per unit time that can be accommodated safely by airspace, when airspace constraints alongwith controller and pilot workload constraints are given" (e.g., special use airspace, convective weather constraints, etc.) [31]. If the air space density reaches at its maximum, there will be no space for adding new routes therefore, it can be stated that air space capacity is maximum. Minimum distance between UAVs' trajectories should be recommended as safe distance.

Total area can be calculated by taking a product of longitudnal and lateral lengths of the airspace and can be presented as:

$$
T A=\sqrt{\left(X_{\max }-X_{\min }\right)^{2} \times\left(Y_{\max }-Y_{\min }\right)^{2}}
$$

$X_{\max }$ and $Y_{\max }$ are the highest where as $X_{\min }$ and $Y_{\min }$ are the lowest points of air space dimentions in longitudnal and lateral axis of cartisian coordinates.

$$
A_{U A V}=\sum_{i=1}^{n} \sum_{k=1}^{S-1}\left(\left|\boldsymbol{x}_{\boldsymbol{i}(\boldsymbol{k}+\mathbf{1})}-\boldsymbol{x}_{\boldsymbol{i k}}\right| \times\left|y_{i(\boldsymbol{k}+\mathbf{1})}-y_{\boldsymbol{i} \boldsymbol{k}}\right|\right)
$$

The area of the airspace occupied by all UAVs with in a specified duration of scenario's flight time which can be written as in (13). Here $\mathrm{x}$ and $\mathrm{y}$ represents the cordinates of UAVs, $\mathrm{s}$ represents the total number of discretised steps of the UAV trajectory and $\mathrm{n}$ represents the number of UAVs in the airspace.

$$
A_{N F Z s}=\sum_{O=1}^{N} A_{O}
$$

$A_{N F Z s}$ represents the area covered by the dynamic and static obstacles during a specific scenario flights. $\mathrm{N}$ is the number of total NFZs in the airspace happened during that interval of time and $A_{O}$ represent the area of respective NFZ which include static and dynamic obstacles.

$$
A_{a v}=T A-A_{U A V}-A_{N F Z s}
$$

By using (12), (13) and (14) the available airspace can be presented as in (15). The number of UAVs which can be catered in the available area are strictly based on the waypoints of UAVs.

\section{- Efficiency}

Efficiency is defined with respect to time or energy. The alternatives leading to good energy efficiency may not lead to good time efficiency[27]. For example, energy efficiency is always maximized by flying through shortest path, but this can result in long ground delays for the vehicle. At other instances, neither time nor energy can be minimized, directly. The ground delay is minimized by selecting first available path found by shortest path algorithm even though it might result in longer path that might arrive late due to delayed start.
Time efficiency is evaluated by calculating the ratio of time taken by UAV during ideal flight to the total time that has been taken by UAV to reach at its destination.

$$
\eta_{\text {time }}=\frac{\Delta t_{\text {ideal }}}{\Delta t_{\text {arrival }}}
$$

In the above equation, an ideal scenario is considered with no weather constraints in airspace. $\Delta t_{\text {ideal }}$ is ideal time taken by any UAV in air during its flight, $\Delta t_{\text {arrival }}$ represents the time taken by UAV to reach at its destination which include ground delays and slow speed.

Energy efficiency is calculated as a ratio of ideal time to the actual time taken by any UAV during its flight in airspace. Mathematically, energy efficiency can be presented as:

$$
\eta_{\text {energy }}=\frac{\Delta t_{\text {ideal }}}{\Delta t_{\text {intheair }}}
$$

$\Delta t_{\text {intheair }}$ is time taken by any UAV in a respective scenario with different weather constraints. Time has been evaluated by considering total distance travelled by UAV at a particular speed (as assumed to be $90 \mathrm{~km} / \mathrm{h}$.) Mathematical definition to find distance travelled by any UAV through all waypoints can be presented as:

$$
\text { Displacement }=\sqrt{\left(x_{2}-x_{1}\right)^{2}+\left(y_{2}-y_{1}\right)^{2}}
$$

Where $x_{2}, y_{2}$ refer to the position coordinates of the $2^{\text {nd }}$ step of $\mathrm{UAV}$, and $x_{1}, y_{1}$ are the values of the previous step.

Total distance is calculated by summing all the displacements at each step of the UAV. Total time taken by any UAV is represented as:

$$
\Delta t_{\text {intheair }}=\frac{\text { Distance }}{\text { Speed }}
$$

More efficient paths will lead to efficiency metrics values close to 1 . As paths become less optimal, their efficiency metrics values will tend toward 0 . The time efficiency is inferior or equal to energy efficiency because, $\Delta t_{\text {arriveal }}>\Delta t_{\text {in The Air }}$ by definition.

\section{Simulation ScEnarios AND Results}

A set of different scenarios are constructed for both type of UAVs by considering different weather conditions, different number of UAVs per scenario, fixed and random start and end locations. Figures of each considered scenario against different parameters are presented below.

\section{A. First scenario of simulations}

During first day scenario, between (9-10 am), 39 rotary-wing UAVs and from (10-11 am), 39 fixed-wing UAVs were not allowed to fly over the seven defined obstacles.This scenario also incorporated the effects of dynamic environment obstacles: adverse rain and winds, and extreme weather conditions. The minimum safe separation distance of $50 \mathrm{~m}$ is used to study the effects on safety of airspace. One of these simulated scenarios is presented in Fig. 2. This scenario is used to evaluate airspace density, capacity and time-energy efficiency. Also, the performance of both type of UAVs are also compared in this scenario. 


\section{B. Second scenario of simulations}

A 29 fixed-wing UAVs and 29 rotary-wing UAVs in airspace are considered without any dynamic environment constraint in this scenario. One of the simulated scenarios is presented in Fig. 3 . This scenario is used as an ideal airspace behavior and is set as a benchmark to evaluate energy and time efficiency.

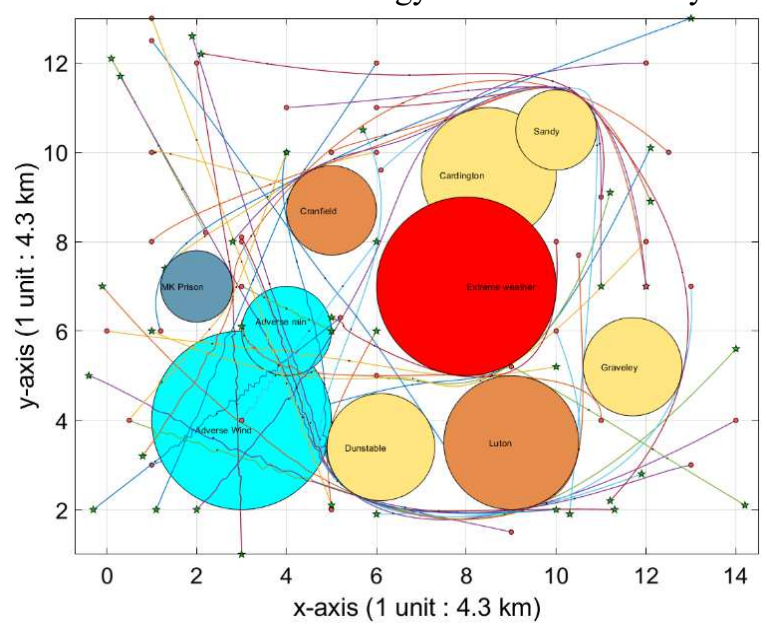

Fig. 2. 39 Rotary-wing UAVs with extreme weather and adverse rain and wind

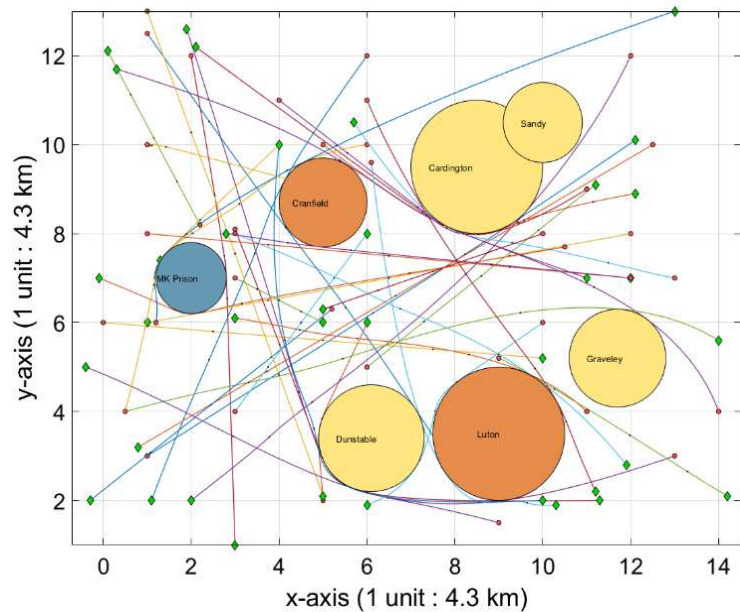

Fig. 3.29 Fixed-wing UAVs without any weather constraints

\section{Third scenario of simulations}

In this scenario 29 fixed wing and 29 rotary-wing UAVs with different weather conditions are used to evaluate UAV safety, density and airspace capacity. One of the simulated scenarios has been presented in Fig. 4.

\section{Fourth scenario of simulation}

In this scenario 29 rotary-wing UAVs are simulated. The waypoints are also prioritized in this scenario with the addition of dummy rows for emergency UAVs in the fixed flight plan. For any emergency UAV service, the high priority levels (1-2) are assigned that results in random way points for emergency UAVs. There were four emergency service UAVS and 25 general purposes fixed location UAVS in this scenario. Also, the dynamic recreational areas are opened in different hourly time zones for more better realistic picture. Different weather constraints are also incorporate to evaluate airspace safety. The simulated scenario with open recreational areas is presented in Fig. 5.

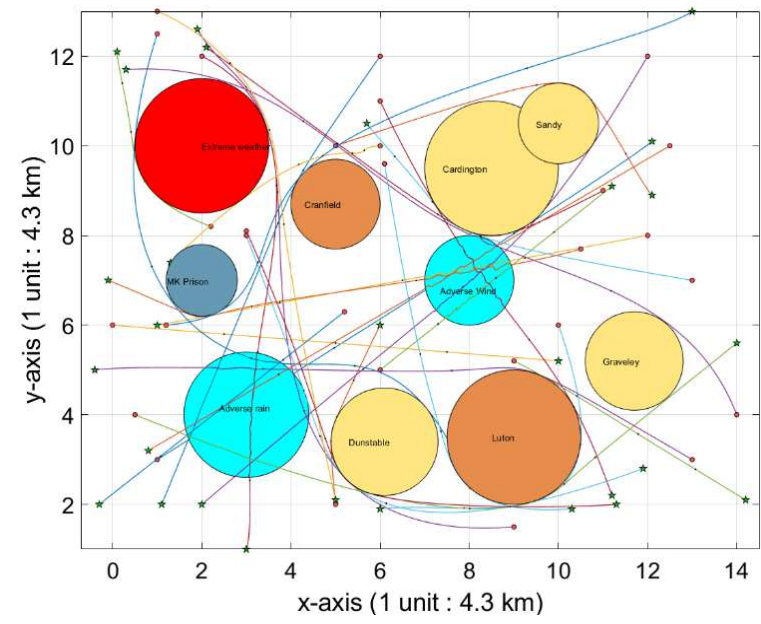

Fig. 429 Rotary-wing UAVs with extreme weather and adverse rain and wind

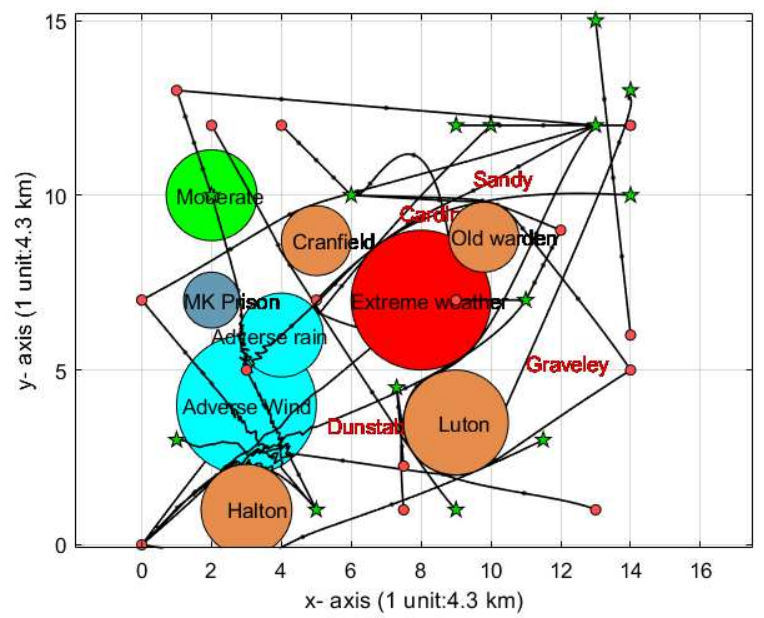

Fig. 5. 29 Rotary-wing UAVs Random emergency with high priority \& open recreational areas

\section{AIRSPACE PERFORMANCE ANALYSIS}

A. Airspace safety

Airspace safety is evaluated using safety metrics discussed in section F-I. A convolution between a sampling cuboid window 1unit $\times 1$ unit $\times 4 \mathrm{~min}$ and whole airspace distribution is conducted to detect the UAVs trajectories in these regions. The detected UAVs trajectories are then discretized into ten equally spaced points. The closeness between trajectories is then calculated by measuring the Euclidian distances and is evaluated among all the possible combinations of trajectories in the sampling window. The measured Euclidian distances are presented as $2 \mathrm{D}$ circles in three-dimensional $\mathrm{x}-\mathrm{y}-\mathrm{t}$ plots as shown in Fig. 6 and Fig. 7 . The radius of these Euclidean distance circles present safety of the airspace. The larger the 
radius of a circle or Euclidian distance in the sampling window, the safer the airspace will be. Three different categories of congestion are designated in green, amber and red colours as in Fig. 6 and Fig. 7 that represent safe, warning and dangerous zones depending upon the radius of the circles.

The results of safety metrics are presented in this section. In order to keep the simulated scenario more realistic, deviations due to weather conditions based on UAVs performance are also considered. The scenarios simulated here incorporate the worst case (with extreme weather, adverse rain, and adverse wind).

The airspace safety analysis of 39 fixed wing UAVs in an airspace environment without weather constraints is presented in Fig. 6. It is observed that the separation distance between UAV trajectories lies in safe zone for most of the congestion points in the airspace. some congestion points are observed at warning level while only a few of the congestion points is designated within the dangerous zone when there is no weather ambiguity.it is also evaluated that about $70 \%$ of the separation distances lie in the safe zone followed by about $25 \%$ at the warning level and only $5 \%$ at the dangerous level.

Analysis of a worst-case scenario with adverse wind, adverse rain and extreme weather ambiguity with $39 \mathrm{UAVs}$ in the airspace is presented in Fig. 7. It has been observed that more dangerous level of congestions is observed in the worst-case scenario as compared to the scenario without dynamic obstacle because UAVs are more deviating under adverse rain, adverse wind and extreme weather conditions that result in a significant loss of separation. It is also evaluated that about $60 \%$ of the separation distances lie in the safe zone followed by about $30 \%$ at the warning level and only $10 \%$ at the dangerous level.

Extreme weather conditions drastically affect the airspace characteristics by making a NFZ for UAVs. Rerouting and congestion are observed in UAVs trajectories that use airspace now occupied by the extreme weather conditions. It is observed that traffic flow patterns are highly influenced by the airborne weather ambiguities, such as adverse wind and adverse rain due to deviation of UAVs from planned trajectories that result in loss of separation distance and cause more dangerous and warning level congestion in airspace.

We also analysed the safety of the airspace in high priority emergencies and open recreational areas scenario in Fig. 8 .It is evaluated in this scenario about $44 \%$ of the Euclidean distancebased circles lie in the danger zone followed by about $29 \%$ at the warning level and only $27 \%$ in the safe zone. This means that random start and finish locations in case of emergencies and opened recreational areas have negatively impact the safety of the airspace.

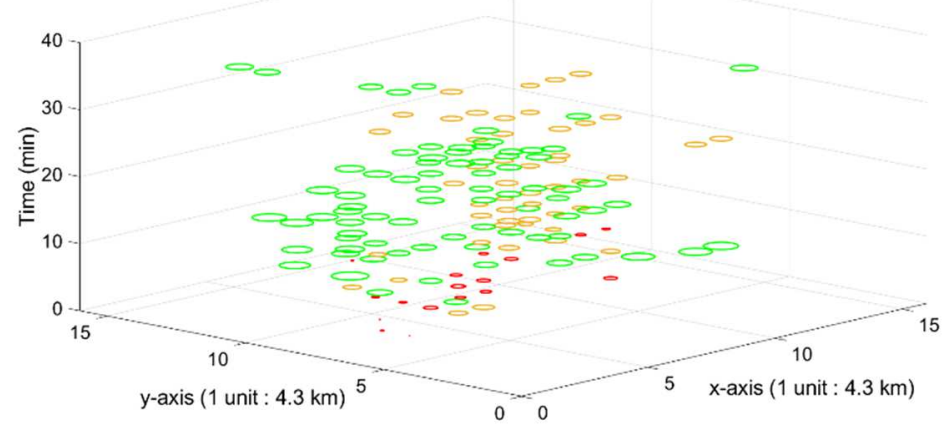

Fig. 6.Safety analysis without weather constraints

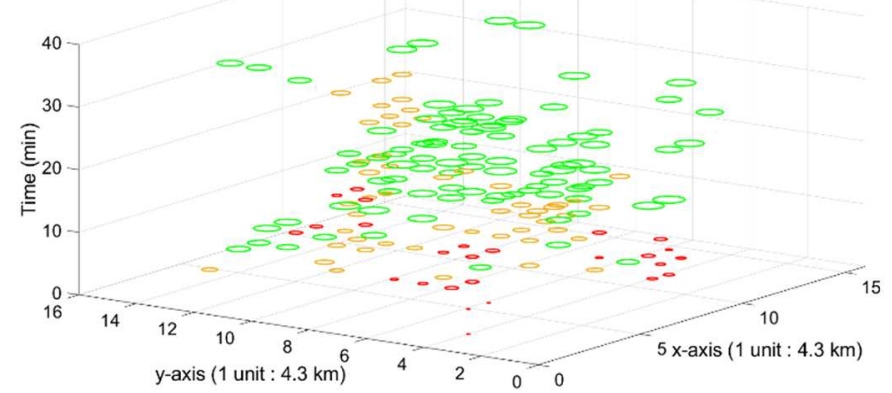

Fig. 7.Safety analysis with adverse wind, adverse rain and extreme weather

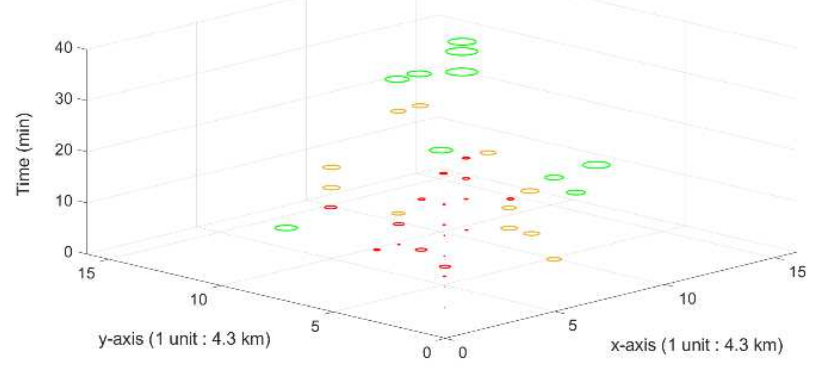

Fig. 8.Safety analysis with random emergencies and open recreational areas

\section{B. Airspace density}

The third scenario of 39 UAVs in airspace has been used to evaluate density metrices. Airspace density has been evaluated by taking a convolution between of UAVs distribution in airspace $\mathrm{d}(\mathrm{x}, \mathrm{y})$ and an all pass filter having a sampling window. $0.5 \mathrm{~km}^{2}$ window of the filter is considered. The whole airspace has been analyzed to measure the density of the UAV trajectories at each square $\mathrm{km}$ area. Low to high density of UAVs per square $\mathrm{km}$ is represented by black to white colors respectively that marks crowded areas. A simulated scenario of 29 rotary-wing UAVs with and without weather constraints as dynamic obstacle has been presented in Fig. 9. Similarly, heatmap of a density for the scenario with extreme weather and adverse rain has been presented in Fig. 10. 


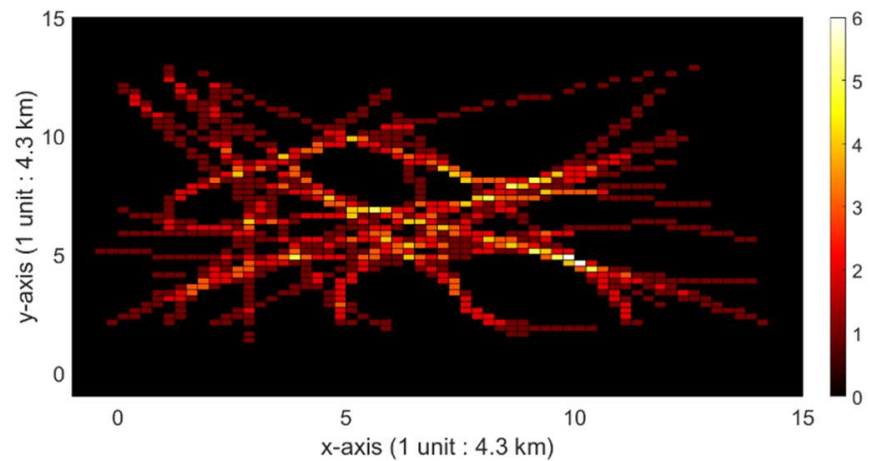

Fig. 9.The heatmap of 29 UAVs airspace density without weather constraints

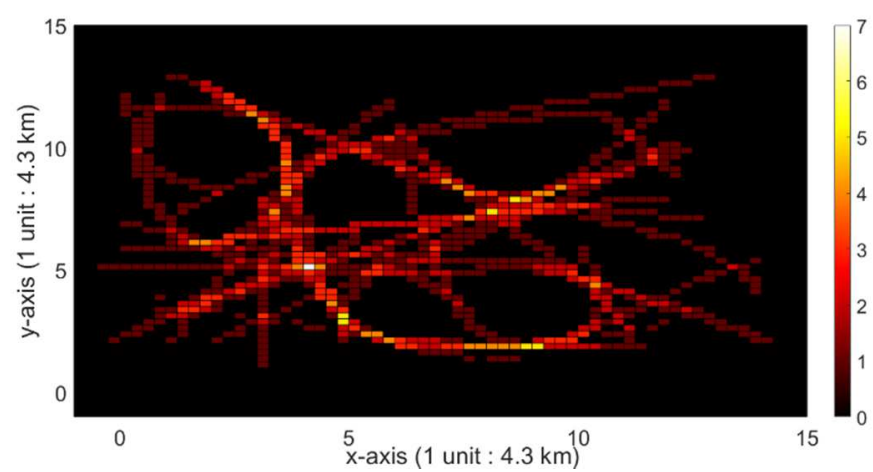

Fig. 10.The heatmap of 29 UAVs airspace density with extreme weather and adverse rain

By the comparison of heatmaps for different scenarios presented in two figures above, it has been observed that the airspace traffic flow patterns are highly influenced by the scenario dynamic parameters. Due to the extreme weather conditions, a large region of airspace has been occupied by the weather that forces the UAVs to avoid the affected region for a safe UTM airspace operation. This result into large deviations in the UAVs waypoints that cause congestion in different regions of an airspace. Airspace density against first simulated scenario of 39 rotary-wing UAVs with extreme weather, adverse wind and rain have been presented in Fig. 11

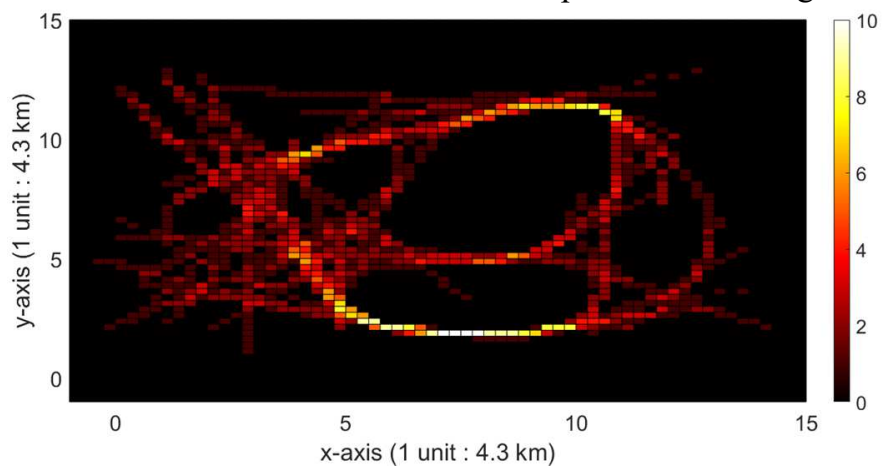

Fig. 11.The heatmap of 39 UAVs airspace density with extreme weather and adverse rain

It has been observed that as the number of UAVs are increased, the density also increases due to large variations in the observed traffic patterns. Moreover, the dynamic weather constraints also play a major role in increasing the density as UAVs trajectory deviates to avoid the weather fronts thus causing variations in air traffic patterns.

\section{Airspace capacity}

To understand airspace capacity with different scenario parameters, the simulation results have been presented in this section. We have used the available area in the airspace to estimate the capacity. The airspace area already occupied is calculated by adding the area of all NFZs (14) and the area used by the UAVs trajectories (13) during flight under different weather conditions. Airspace structure with all the discussed scenarios is presented in Fig. 12.

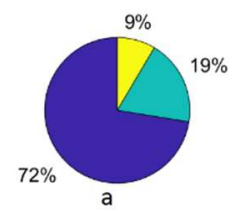

$8 \%$

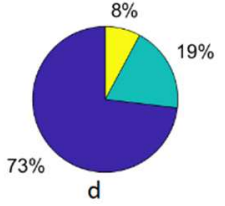

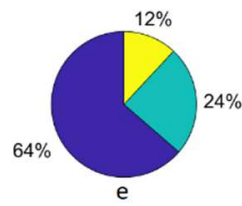

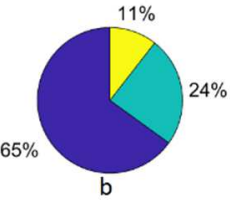

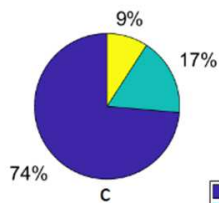

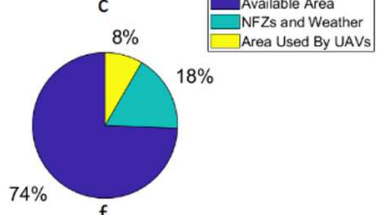

Fig. 12. a) 29 fixed wing UAVs with extreme weather, adverse rain and adverse wind. b) 39 fixed wing UAVs with extreme weather adverse rain and adverse wind. c) 39 fixed wing UAVs without dynamic constraints. d) 29 rotary-wing UAVs with extreme weather adverse wind and adverse rain. e) 39 rotary-wing UAVs with extreme weather adverse wind and adverse rain. f) 29 rotary-wing UAVs without any dynamic constraint.

It is observed in Fig. 12 that in the absence of dynamic weather conditions least area is occupied. Thus, there is less congestion in the airspace. A small increase in the occupied area has been observed with adverse wind and adverse rain because of the deviation in the UAVs trajectories which are passing through these regions. Whereas a significant amount of increase in the occupied area has been observed with extreme weather as an environmental constraint because UAVs are not allowed to pass through extreme weather conditions to avoid any conflict creating weather fronts. The available airspace region has been evaluated by subtracting the occupied area from the total area of current airspace.

Fig. 12 has been scaled with a ratio of $1: 4.3 \mathrm{~km}$. If UTM is required to accommodate more UAVs in airspace and cater increased density, a proper estimation of airspace capacity is required. The available area in the airspace is the best way to estimate the room for new coming emergency or planned UAVs. The presented available area shows the variation of available airspace with respect to different scenario parameters. It has been observed from the different scenarios that traffic flow patterns are highly affected by different weather conditions due to rerouting in case of extreme weather. There is more congestion in airspace as less areas are available due to extreme weather conditions. Thus, the UAVs are taking larger turns to reach the destination by avoiding any airborne extreme weather constraint. It is observed that as the number of UAVs are increased the occupied airspace is also increased slightly by $3-4 \%$ under extreme weather conditions. this increase is due to 
large variations in the airspace traffic patterns as seen in Fig. 12 $\mathrm{b}$ and $\mathrm{e}$.

\section{Airspace energy and time efficency}

The time efficiency of each UAV flight has been evaluated by using its ideal time. The time efficiency of both scenarios has been presented in Fig. 13 and Fig. 14 given below:

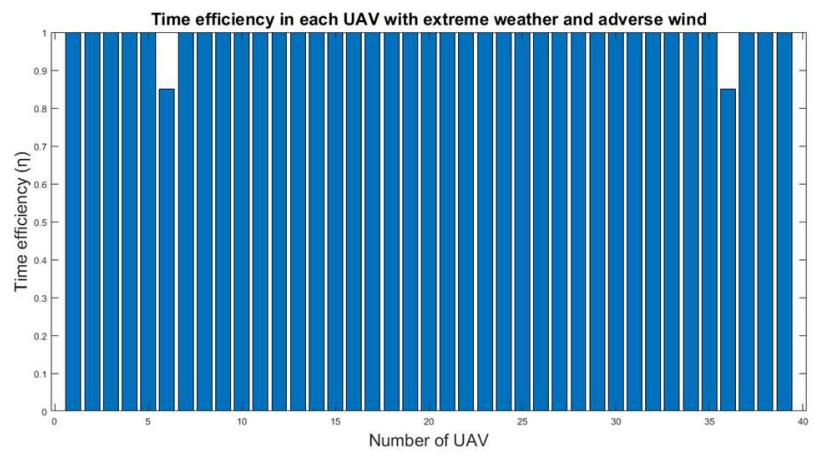

Fig. 13.Time efficiency of UAV with extreme weather and adverse wind

The decrease in the time efficiency of the UAVs with a low priority that are subjected to ground delay. Similar to energy efficiency, extreme weather has an impact on time efficiency due to the unavailability of airspace regions and the changes in traffic flow patterns, forcing low priority UAVs to ground delay.

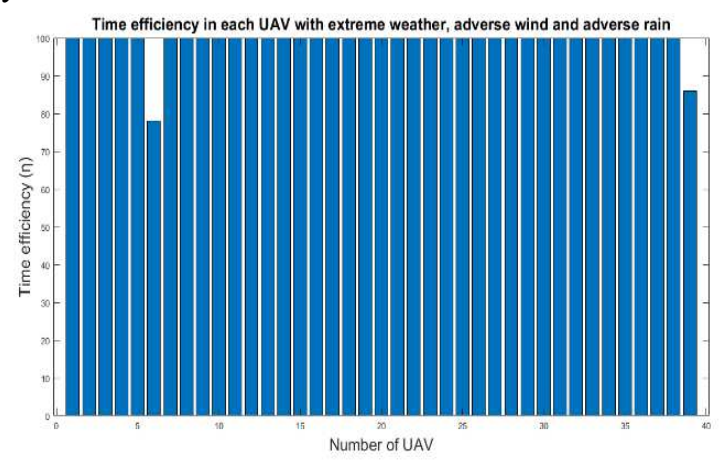

Fig. 14.Time efficiency in extreme weather, adverse wind and adverse rain

The evaluated energy efficiency of each UAV with a certain scenario has been presented in Table 3. The energy efficiency of the airspace is evaluated by comparing battery usage and thus cost incurred by UAVs during the flight time in comparison with the ideal case scenario when there are no weather constraints. In this context, it is observed from Fig. 2 above that as large number of UAVs are flying in the adverse wind scenario, there observed a slightly higher loss of energy efficiency in rotary-wing UAVs due to their dynamic constraints.

\begin{tabular}{|l|c|c|c|}
\hline Scenario description & UAV type & $\begin{array}{c}\text { Number } \\
\text { of UAV }\end{array}$ & $\begin{array}{c}\text { Energy } \\
\text { efficiency }\end{array}$ \\
\hline \multirow{3}{*}{$\begin{array}{l}\text { Eextreme weather, } \\
\text { adverse rain and } \\
\text { adverse wind }\end{array}$} & Rotary-wing & 29 & $96 \%$ \\
\cline { 2 - 4 } & Fixed-wing & 29 & $98 \%$ \\
\cline { 2 - 4 } & Rotary-wing & 39 & $89 \%$ \\
\cline { 2 - 4 } & Fixed-wing & 39 & $91 \%$ \\
\hline
\end{tabular}

Table 3:The evaluated efficiency of airspace for different scenarios

\section{E. Characterization of traffic flow patterns}

The characterization of the UAVs traffic flows in Bedfordshire has been done by conducting safety, density, capacity and efficiency analysis for both time and energy as presented in above sections. Historical patterns were taken from different time intervals of the day to capture the variability in weather conditions considered as weather constraints. It is observed from safety analysis that traffic flow patterns in Bedfordshire airspace have been changed due to trajectory deviations that are caused by weather constraints such as adverse winds, rain and extreme weather conditions thus threating the safety of airspace.

The density map reveals that adverse weather conditions and specifically extreme weather conditions result in congestion patterns and also create some void holes resulting in reduced capacities as discussed in section V- B above. It can be seen from the heat map that yellow highlighted trends show the congested areas. The North-West and South-West regions in $15 \times 15(1: 4.3 \mathrm{~km})$ area of Bedfordshire are denser due to increased density of trajectory points spatially. The capacity analysis reveals that there is almost $8 \%$ reduction (Fig. 12(b and e) in available airspace area in case of extreme weather as compared to absence of any dynamic obstacle. This reduction in the airspace is due to trajectory deviations because of weather front causing void holes to be created as trajectory patterns and the UAVs are taking larger turns to reach the destination by avoiding such dynamic weather constraints. The energy efficiency analysis reveals that accumulated airspace energy efficiency shows almost $7 \%$ reduction when extreme weather conditions are met. Also, the time efficiencies have been disrupted in such adverse conditions due to either rerouting or ground delay implementations. It has been observed that time delay of the order of 1.8 to 2.5 minutes are experienced in this deconfliction process thus affecting the time efficiencies This may adversely affect the customer satisfaction and increase the operational costs. The extreme weather dynamic obstacle cloud has completely deviated the paths of both type of UAVs that is evident in comparison of Fig. 2 and Fig. 4. The void area between Cardington, Luton and Dunstable is accommodating a number of trajectory points that became unusable due to evolution of extreme weather cloud in this region resulting in congestion due to rerouting. All emergency services UAVs have been assigned with level 1 priority. These emergency UAVs leads to ground delays and rerouting for some low priority UAVs causing reduction in their time and energy efficiency, as presented in Fig. 13 and Fig. 14. It has also been observed that random trajectories of these emergency UAVs and removal of restriction by weather constraints such as recreational areas have adversely affect the safety of the airspace. It has been observed from analysis that traffic flow patterns in Bedfordshire airspace changed due to variability in weather constraints causing a change in airspace characteristics and patterns. It is thus concluded that dynamic weather and UAVs emergency constraints play a significant role in defining various traffic flow behaviors such as unusable closed spaces and congestion. 


\section{CONCLUSION \& Future RECOMMENDATIONS}

The current work has formulated a customized simulation framework to characterize traffic flow behaviors using safety, capacity, and efficiency related metrics. The safety analysis that was conducted by evaluating the separation distance of UAVs in spatial-temporal scale. This separation distance can be executed at the planning stage while designing the UAV trajectories and later adopting see and avoid philosophy in realtime to enforce this safety envelope using on-board sensors. Moreover, the path optimization of the UAV's can be useful in minimizing the flying cost and efficiency of UAVs. The ML algorithms are highly supportive and efficient in solving optimization problems where there are many variable factors and enormous amount of information is involved. In addition to that, path optimization of the UAVs through ML algorithms also considers other factors such as authorized airspace restrictions, other aircraft paths in the planning operations to maintain the safety of the UAV, other aircrafts, ground pedestrians and properties [32]. We therefore suggest a deep learning model by integrating Reinforcement learning (RL) and A deep neural network (DNN) algorithms for optimizing the path policy in-order to maximize the energy efficiency of UAVs.

\section{REFERNCES}

[1] M. Intelligence, "DRONES MARKET - GROWTH, TRENDS, COVID-19 IMPACT, AND FORECASTS (2021 - 2026).pdf.” 2020.

[2] P. Kopardekar, J. Rios, T. Prevot, M. Johnson, J. Jung, and J. E. Robinson, "Unmanned aircraft system traffic management (UTM) concept of operations," 16th AIAA Aviat. Technol. Integr. Oper. Conf., pp. 1-16, 2016.

[3] E. Mueller, P. Kopardekar, and K. Goodrich, "Enabling airspace integration for high-density on-demand mobility operations," 17th AIAA Aviat. Technol. Integr. Oper. Conf. 2017, no. June, pp. 1-24, 2017, doi: $10.2514 / 6.2017-3086$

[4] M. Xue, "Sensitivity analysis of key factors in high density unmanned aerial system operations," 2020. doi: 10.2514/6.20190688 .

[5] M. Bulusu, V., Sengupta, R., Mueller, E. R., and Xue, "A throughputbased capacity metric for low-altitude airspace," 2020. doi: 10.2514/6.2018-3032.

[6] M. C. R. Murça, M. X. Guterres, M. de Oliveira, J. B. Tarelho Szenczuk, and W. S. S. anna Souza, "Characterizing the Brazilian airspace structure and air traffic performance via trajectory data analytics," J. Air Transp. Manag., vol. 85, no. November 2019, 2020, doi: 10.1016/j.jairtraman.2020.101798.

[7] X. Oliver, L. Basora, B. Viry, R. Alligier, X. Olive, and B. Viry, "Deep Trajectory Clustering with Autoencoders," 2020, [Online]. Available: https://hal-enac.archives-ouvertes.fr/hal-02916241.

[8] Z. Sándor, "Challenges caused by the unmanned aerial vehicle in the air traffic management," Period. Polytech. Transp. Eng., vol. 47, no. 2, pp. 96-105, 2019, doi: 10.3311/PPtr.11204.

[9] S. Bijjahalli, R. Sabatini, and A. Gardi, "Advances in intelligent and autonomous navigation systems for small UAS," Prog. Aerosp. Sci., vol. 115, no. June, p. 100617, 2020, doi: 10.1016/j.paerosci.2020.100617.

[10] P. N. Tran, D. T. Pham, S. Kuan Goh, S. Alam, and V. Duong, "An interactive conflict solver for learning air traffic conflict resolutions," J. Aerosp. Inf. Syst., vol. 17, no. 6, pp. 271-277, 2020, doi: 10.2514/1.I010807.

[11] A. Carrio, C. Sampedro, A. Rodriguez-Ramos, and P. Campoy, “A review of deep learning methods and applications for unmanned aerial vehicles," J. Sensors, vol. 2017, 2017, doi: 10.1155/2017/3296874.

[12] P. Kopardekar, "Enabling civilian low-altitude airspace and
Unmanned Aerial System (UAS) operations by Unmanned Aerial System Traffic Management (UTM)," AUVSI Unmanned Syst. 2014, vol. 2, no. April 2014, pp. 1678-1683, 2014.

[13] H. X. Chen, Y. Nan, and Y. Yang, "Real-time conflict resolution algorithm for multi-UAV based on model predict control," Algorithms, vol. 12, no. 2, 2019, doi: 10.3390/a12020047.

[14] European Commission, "Commission Implementing Regulation (EU) 2019/947 of 24 May 2019 on the rules and procedures for the operation of unmanned aircraft," $O J$, vol. L, no. 152, pp. 45-71, 2019, [Online]. Available: https://eurlex.europa.eu/eli/reg impl/2019/947/oj.

[15] A. P. Soares, "EXPLORING THE RANGE OF WEATHER IMPACTS ON UAS OPERATIONS," J. Chem. Inf. Model., vol. 53, no. 9, pp. 1689-1699, 2013.

[16] X. Olive and L. Basora, "Detection and identification of significant events in historical aircraft trajectory data," Transp. Res. Part C, vol. 119, no. August, p. 102737, 2020, doi: 10.1016/j.trc.2020.102737.

[17] J. Oehling and D. J. Barry, "Using machine learning methods in airline flight data monitoring to generate new operational safety knowledge from existing data," Saf. Sci., vol. 114, no. January, pp. 89-104, 2019, doi: 10.1016/j.ssci.2018.12.018.

[18] J. J. Roldan, J. Del Cerro, and A. Barrientos, "A proposal of methodology for multi-UAV mission modeling," 2015 23rd Mediterr. Conf. Control Autom. MED 2015 - Conf. Proc., pp. 1-7, 2015, doi: 10.1109/MED.2015.7158721.

[19] C. A. O. Longbing, "Data Science: A Comprehensive Overview," arXiv, vol. 50, no. 3, 2020

[20] C. Büsing, D. Kadatz, and C. Cleophas, "Capacity uncertainty in airline revenue management: Models, algorithms, and computations," Transp. Sci., vol. 53, no. 2, pp. 383-400, 2019, doi: $10.1287 /$ trsc. 2018.0829 .

[21] S. H. Chung, H. L. Ma, and H. K. Chan, "Cascading Delay Risk of Airline Workforce Deployments with Crew Pairing and Schedule Optimization," Risk Anal., vol. 37, no. 8, pp. 1443-1458, 2017, doi: 10.1111/risa.12746.

[22] E. Capello, G. Guglieri, and F. Quagliotti, A waypoint-based guidance algorithm for mini UAVs, vol. 2, no. PART 1. IFAC, 2013.

[23] M. S. Y. Hii, P. Courtney, and P. G. Royall, "An evaluation of the delivery of medicines using drones," Drones, vol. 3, no. 3, pp. 1-20, 2019, doi: 10.3390/drones3030052.

[24] I. Koohi and V. Z. Groza, "Optimizing Particle Swarm Optimization algorithm," Can. Conf. Electr. Comput. Eng., pp. 1-5, 2014, doi: 10.1109/CCECE.2014.6901057.

[25] D. Wang, D. Tan, and L. Liu, "Particle swarm optimization algorithm: an overview," Soft Comput., vol. 22, no. 2, pp. 387-408, 2018, doi: 10.1007/s00500-016-2474-6.

[26] A. Alharbi, A. Poujade, K. Malandrakis, I. Petrunin, D. Panagiotakopoulos, and A. Tsourdos, "Rule-based conflict management for unmanned traffic management scenarios," AIAA/IEEE Digit. Avion. Syst. Conf. - Proc., vol. 2020-Octob, 2020, doi: 10.1109/DASC50938.2020.9256690.

[27] M. I. I. Reports, "Development of a framework to compare lowaltitude unmanned air traffic management systems," 2020. doi: $10.2514 / 6.2021-0812$.

[28] R. Golding, "Metrics to characterize dense airspace traffic," no. June, 2018.

[29] M. Ribeiro, J. Ellerbroek, and J. Hoekstra, "Analysis of conflict resolution methods for manned and unmanned aviation using fasttime simulations," SESAR Innov. Days, no. December, 2019.

[30] Z. Zhao et al., "A simulation framework for fast design space exploration of unmanned air system traffic management policies," arXiv, 2019.

[31] J. Krozel, J. S. B. Mitchell, V. Polishchuk, and J. Prete, "Maximum Flow Rates for Capacity Estimation in Level Flight with Convective Weather Constraints," Air Traffic Control Q., vol. 15, no. 3, pp. 209238, 2007, doi: 10.2514/atcq.15.3.209.

[32] J. Qiu, Q. Wu, G. Ding, Y. Xu, and S. Feng, "A survey of machine learning for big data processing," EURASIP J. Adv. Signal Process., vol. 2016, no. 1, 2016, doi: 10.1186/s13634-016-0355-x. 
$2021-11-15$

\section{Identification and characterization of traffic flow patterns for UTM application}

Alharbi, Abdulrahman

IEEE

Alharbi A, Petrunin I, Panagiotakopoulos D. (2021) Identification and characterization of traffic flow patterns for UTM application. In: 2021 AIAA/IEEE 40th Digital Avionics Systems Conference (DASC), 3-7 October 2021, San Antonio, USA https://doi.org/10.1109/DASC52595.2021.9594494

Downloaded from Cranfield Library Services E-Repository 